\title{
Migraciones internacionales en Canarias: Geografía, academia y praxis
}

\author{
Josefina Domínguez MujICA \\ Departamento de Geografía \\ Universidad de Las Palmas de Gran Canaria \\ jdominguez@dgeo.ulpgc.es \\ Vicente Manuel Zapata HernándeZ \\ Departamento de Geografía \\ Universidad de La Laguna \\ vzapata@ull.es
}

Recibido: 19 de diciembre de 2011

Enviado a evaluar: 30 de marzo de 2012

Aceptado: 24 de abril de 2012

\section{RESUMEN}

En pocas ocasiones se brinda a los geógrafos la posibilidad de que sus investigaciones orienten las actuaciones políticas, conjugando academia y praxis. Por ello, la firma de un convenio entre el Parlamento de Canarias y las dos universidades del archipiélago, creando un grupo de expertos y una subcomisión parlamentaria para orientar las actuaciones políticas en la gestión de la inmigración, representó un hito en la acción de dichas instituciones. Para los investigadores, la oportunidad de poner en práctica una disciplina de profunda repercusión social. Para los parlamentarios, la posibilidad de fundamentar sus actuaciones legislativas en un basamento científica y socialmente acreditado. El análisis de esta iniciativa y del trabajo efectuado desde la perspectiva geográfica constituye el objeto de este artículo, planteado desde el convencimiento de que puede guiar otras iniciativas semejantes en las que ciencia y compromiso social estrechen sus vínculos, especialmente en el ámbito de las migraciones internacionales.

Palabras clave: geografía aplicada, geodemografía, migraciones, praxis, Canarias.

\section{International Migrations in the Canary Islands: Geography, Academia and Praxis}

\begin{abstract}
Only seldom do geographers experience the possibility of effectively providing guidelines for policy makers, of bridging the gap that usually separates academia from praxis. The agreement signed between the Parliament of the Canary Islands and the Islands' two universities, setting up a group of experts and a parliamentary subcommittee in order to provide guidance for immigration policy-making, has been a landmark in the case of both academic institutions. The agreement has provided researchers with the opportunity to put an applied knowledge of widespread social impact into practice. Likewise, Members of Parliament have been offered the chance to base their policy-making on sound social and scientific foundations. The present paper analyses this initiative and the work undertaken from a geographical
\end{abstract}


perspective, based on the authors' conviction that it might help other similar institutions to bring science and social commitment closer together, especially within the context of international migrations.

Key words: Applied Geography, Geodemography, Migrations, Praxis, Canary Islands.

\section{Migrations internationales aux Canaries}

\section{RÉSUMÉ}

Il est rarement offert aux géographes la possibilité d'orienter les actions politiques par leurs recherches en conjuguant académie et praxis. Or, la signature d'une convention entre le Parlement des Canaries et les deux universités de l'archipel, entraînant la création d'un groupe d'experts et d'une sous-commission parlementaire pour orienter les interventions politiques dans la gestion de l'immigration, a marqué un tournant dans l'action de ces institutions. Les chercheurs ont eu l'occasion de mettre en pratique une discipline d'une profonde répercussion sociale, et les parlementaires la possibilité d'asseoir leurs décisions législatives sur une base scientifiquement et socialement reconnue. Le but de cet article est d'analyser cette initiative et le travail effectué du point de vue géographique, dans la certitude qu'il peut servir de guide à d'autres initiatives semblables dans lesquelles la science et l'engagement social puissent resserrer leurs liens, en particulier dans le domaine des migrations internationales.

Mots-clés: géographie appliquée, géodémographie, migrations, praxis, Canaries.

\section{INTRODUCCIÓN}

En la coyuntura económica expansiva de los años 1995 a 2007 se produjo un notable incremento de la atracción inmigratoria de Canarias, a consecuencia de una serie de factores, entre los que destacan el proceso de internacionalización de la economía regional, la condición del archipiélago como frontera exterior de la Unión Europea, los históricos vínculos de las islas con los continentes africano y americano, así como su intenso proceso de desarrollo turístico. A lo largo de ese mismo periodo se consolidaron en las dos universidades canarias los estudios de Geografía de la Población en la temática específica de los procesos migratorios internacionales. Se realizaron tesis doctorales en esta materia y se publicó un número considerable de trabajos, que alcanzaron una importante difusión en el ámbito académico. En el año 2001 se creó además el Observatorio de la Inmigración de Tenerife (OBITen), una iniciativa conjunta del Cabildo de Tenerife y la Universidad de La Laguna, producto de las conclusiones de una investigación académica de carácter geográfico.

Por otra parte, los geógrafos estuvieron representados en el comité de expertos que creó el Gobierno de Canarias en el año 2002, como responsables del estudio sociodemográfico sobre Población e Inmigración en Canarias. En el año 2006, cuando se incrementó la presión marítima irregular de personas indocumentadas procedentes de Mauritania, Senegal, Gambia, Guinea Bissau, entre otras áreas de origen, conocida a partir de entonces como crisis de los cayucos, ante la multiplicación de voces de alarma y de mensajes xenófobos, los geógrafos sumaron sus voces a las de economistas, sociólogos, juristas y otros científicos sociales, difundiendo un manifiesto que concluía con las siguientes palabras: "invitamos a la reflexión acerca de cómo los 
retos que plantea la inmigración se pueden convertir en oportunidades, y además, animamos a que se estudien con rigor los efectos de la inmigración antes de condenarla por el mero hecho de contribuir a la generación de cambios más o menos intensos en diversos planos de nuestra realidad"1. También desde la primera fecha citada, geógrafos de ambas universidades canarias representan a ambas instituciones en el Foro Canario de la Inmigración.

Desde la profunda convicción de que, parafraseando a Daniel Hiernaux, "la geografía tiene en sus manos algunas claves para construir otro futuro, para humanizar el mundo" (Hiernaux, 2010, 59), los geógrafos que han trabajado en la temática de las migraciones en Canarias adquirieron un postrer compromiso, cuando formaron parte del grupo de trabajo que se creó como fruto de un acuerdo con el Parlamento de Canarias para prestarle asesoramiento en esta materia ${ }^{2}$. Dicha tarea, en la que participaron coordinando un amplio equipo integrado por investigadores de las dos universidades canarias, adscritos a los ámbitos de conocimiento de la economía, sociología, ciencias jurídicas y ciencias de la educación, concluyó con la presentación de un informe sintético y propositivo que diseña una cierta hoja de ruta en la gestión de la inmigración en el archipiélago ${ }^{3}$.

Creemos que este informe constituye un buen ejemplo del necesario acercamiento de la academia a la praxis política. Desde nuestro punto de vista, se cumplieron aquellos requisitos que enunciaba Michael Pacione, cuando decía que los geógrafos debían valerse de la geografía aplicada como un conocimiento útil al desafío que representa la mejora de las condiciones de vida del presente y del futuro (Paccione, 1999) o bien Horacio Capel, cuando afirmaba que el geógrafo debe estudiar y describir el mundo a partir de teorías, contribuir a su comprensión e intentar cambiarlo, pero para ello lo primero es la investigación y la acción ciudadana (Capel, 2001). Sólo en estos términos cabe interpretar el papel asumido por la geografía junto a otras ciencias sociales en la sesión parlamentaria en la que todos los grupos políticos representados en la Cámara autonómica de Canarias aprobaron un texto riguroso y propositivo, que había nacido del intercambio de opiniones y pareceres entre los investigadores y los representantes públicos.

El tema de las migraciones internacionales se convierte, una vez más, en punto de encuentro científico para el trabajo interdisciplinar en el ámbito de las ciencias sociales, revelando sus diversas facetas la necesidad de una aproximación multifocal. Su comprensión integral, como fenómeno complejo, y la de sus implicaciones, sólo se consiguen, habitualmente, gracias a la formación de equipos de trabajo que combinen distintas perspectivas y métodos de análisis, sobre todo cuando se pretende alcanzar

${ }^{1}$ El País, 13 de noviembre de 2006, 17.

${ }^{2}$ Convenio de colaboración entre el Parlamento de Canarias y las Universidades de La Laguna y de Las Palmas de Gran Canaria para la elaboración de un estudio sobre la inmigración en Canarias y para la prestación de asesoramiento en la realización de actividades en dicho ámbito, suscrito en Santa Cruz de Tenerife el 14 de abril de 2008, con una vigencia entre los años 2008 y 2010.

${ }^{3}$ Informe de la Subcomisión aprobado por la Comisión 7L/AGSC-0001 Sobre la situación de la inmigración en Canarias (Boletín Oficial del Parlamento de Canarias, número 17, 14/01/2011). http:// www.parcan.es/pub/bop/71/2011/017/bo017.pdf 
resultados con carácter aplicado en contextos que han observado una significativa expansión de la movilidad. En ellos, la Geografía suele aportar su dimensión de amplia comprensión de procesos socio-territoriales, su capacidad de análisis y síntesis de información de diferente naturaleza, así como su creciente vocación de influir con propuestas concretas en la mejora de la realidad de cada lugar.

En sintonía con ese trabajo, los objetivos que nos trazamos en este artículo son los de reflexionar acerca de la función aplicada de la Geografía de la Población, caracterizar la metodología de trabajo desarrollada por el grupo de expertos y la subcomisión parlamentaria responsables del informe, así como defender las conclusiones alcanzadas, como propias de un estudio profundo que combina acertadamente un sólido basamento teórico, la interdisciplinariedad con otras ciencias sociales y la praxis política. De esta forma, consideramos que se puede contribuir al avance de la ciencia, a la crítica, al compromiso social y, a la vez, a la dignificación de la profesión de geógrafos, porque, tal y como dice Jacques Lévy, "el papel de los geógrafos no es el de refutar el derecho de los ciudadanos a decidir, sino el de mostrarles las consecuencias últimas de sus decisiones" (Lévy, 2011, 90).

\section{MARCO CONCEPTUAL: LA DIMENSIÓN APLICADA DE LA GEOGRA- FÍA DE LA POBLACIÓN}

En un artículo publicado en la revista Population, Space and Place, David A. Plane auguraba en 2004 un futuro en el que las nuevas metodologías y fuentes de datos demográficos conducirían a un papel más destacado de la investigación geográfica de la población en el ámbito gubernamental (Plane, 2004). Es cierto que esta idea pivotaba ya en los trabajos de geografía de la población que se realizaron desde los años sesenta, setenta y primeros ochenta del siglo XX, trabajos señeros, cuyos autores alcanzaron un gran reconocimiento en la disciplina (Pierre George, 1965; Jacqueline Beaujeu-Garnier, 1966; Wilbur Zelinsky, 1966; George Demko, M. Rose Arnold \& George Schnell, 1970; Gary Peters \& Robert Larkin, 1979; Daniel Noin, 1979; John Clarke, 1981; Huw Jones, 1981; John Clarke, 1984) Sin embargo, en esas fechas, la investigación en este campo estaba estrechamente vinculada a grandes pautas, lo que podríamos identificar como macromagnitudes demográficas, y a la relación que ello guardaba con el desarrollo de los recursos y con las políticas demográficas que podían limitar el crecimiento de la población, teniendo un gran peso aún el debate maltusiano. Así lo demuestran, también, las conferencias mundiales sobre población, un magnífico ejemplo de la íntima relación que se otorgaba al binomio: demografíaeconomía.

Es necesario que transcurran algunos años para que los análisis de demografía espacial, lo que denominan en el ámbito anglosajón como geodemografía ${ }^{4}$, así como

${ }^{4}$ En España se utilizan indistintamente los términos geografía de la población y geodemografía. Sin embargo, en el ámbito anglosajón, geodemografía es un concepto utilizado para caracterizar pequeñas áreas (vecindarios) que presentan semejanzas o diferencias en cuanto a las formas de vida y comportamiento de sus poblaciones, lo que permite trasladarles diferenciados productos, servicios y medios. 
la utilización de nuevas metodologías de investigación (análisis longitudinales, sistemas de información geodemográfica, etc.), promueva otras miradas, otras perspectivas en el estudio de la población, en sintonía con la geografía social y con la teoría social. Por ello, desde la década de los noventa se alzan nuevas voces que defienden la necesidad de que los geógrafos de la población más que describir patrones, deberían estar resueltos a identificar problemáticas demográficas concretas y sus repercusiones espaciales (Findley, 1993; Nash, 1994). En palabras de Paul White y Peter Jackson "with a recognition of the power of arguments from social theory, population geography could be reconnected to human geography as a whole, strengthening its ability to research major contemporary issues" (White \& Jackson, 1995, 111).

En España, por esos mismos años, se insistía en la necesidad de conducir los estudios de población hacia una dimensión aplicada. Así, en 1991, Vicente Gozálvez afirmaba que las investigaciones en esa vertiente de la geodemografía se reforzarían en el futuro y que "las migraciones continuarían siendo uno de los temas más tratados, en razón de las consecuencias demográficas, económicas y sociales que estos procesos implican" (Gozálvez, 1991, 218). Siete años después, los logros no debieron ser tan importantes cuando el mismo autor reconoce que, en palabras de Patrick Festy, director del I.N.E.D., los poderes públicos muestran una curiosidad más bien escasa respecto a los problemas demográficos, y que, por ello, la información geodemográfica debía situarse en el centro de las decisiones de los agentes sociales. "La geografía de la población no puede escapar a la doble tarea de ser una ciencia pura y una ciencia aplicada, es decir, una ciencia política en el sentido pleno del término, porque puede contribuir a una mejor gestión de los asuntos públicos" (Gozálvez, 1998, 26).

En esta misma línea de pensamiento se expresaba Julio Vinuesa en 1995, cuando afirmaba que, a pesar del gran desarrollo de la geografía de la población en España, seguía siendo insuficiente la relevancia que venía desempeñando el análisis geodemográfico en la actividad planificadora (Vinuesa, 1995, 147). A esta circunstancia, tanto este autor, como otros, oponían la necesidad de que la geodemografía asumiera un nuevo papel, el de describir con claridad los problemas, mostrando con rigor científico las tendencias demográficas y sus consecuencias, lo que en términos de planificación territorial identificaba Eugenio Burriel como proponer esquemas tendenciales y mostrar que la evolución futura dependerá de decisiones políticas (2002). O en otras palabras, "quizá el principal reto de la geografía de la población sea pasar de explicar los componentes de una población en el territorio a un debate más complejo sobre lo que significa el espacio, cómo los espacios se viven, construyen y representan, dependiendo de las características sociodemográficas de poblaciones o subpoblaciones diferenciadas" (Mendoza, 2010, 17).

Estas premisas conceptuales guiaron el análisis, la consulta y el debate acerca del informe sobre la situación de la inmigración en Canarias desde la perspectiva geográfica. La caracterización de este proceso y de sus distintas facetas, la identificación de sus dinámicas y de sus implicaciones, así como la reflexión sobre las medidas adoptadas y las nuevas propuestas, ante los problemas detectados, trataron de arrojar luz en un panorama en el que "realidad e imagen, cifras y percepción, hechos y opiniones, efectos y causas... lejos de ajustarse se confunden, se contraponen, se contradicen" 
(Reques, 2011, 47). En consecuencia, los geógrafos y los demás científicos sociales que integraron el equipo de trabajo trataron de romper tópicos infundados, de hacer distinguir entre realidades y mitos, entre hechos y opiniones, entre ciencia social e ideología, tejiendo una profunda interrelación con los representantes parlamentarios, para plantear recomendaciones que podían beneficiar la toma de decisiones en materia migratoria. Por ello, las propuestas contenidas en el informe, que nacen de un profundo compromiso social, sólo alcanzarán éxito cuando el trabajo desarrollado se traduzca en directrices que deriven en actuaciones específicas. Con el concurso de la praxis política, se logrará que la gestión de la inmigración adquiera el protagonismo que ha tenido y sigue teniendo en la nueva realidad social de Canarias.

\section{EL ESTUDIO DE LAS MIGRACIONES INTERNACIONALES EN CANARIAS DESDE UNA PERSPECTIVA APLICADA}

La dimensión aplicada del análisis que dio pie a la elaboración de los informes particulares que constituían el objeto de investigación y su presentación ordenada para el debate con los miembros de la subcomisión parlamentaria, determinaron la elección de las temáticas y la composición de cada uno de ellos. La finalidad del trabajo condicionó el formato escogido, tanto en lo que respecta al contenido como en lo que se refiere a su extensión y a su estructura, dado que se trataba de garantizar la efectividad de las tareas subsiguientes, es decir, el debate, el intercambio de pareceres y la asunción de las conclusiones de dichos informes en el marco parlamentario. De todos es sabido que la labor legislativa entraña una tarea de información exhaustiva por parte de los miembros de cualquier órgano de representación democrática y que, además, el tiempo es un valor preciado y escaso. Por ello, cuando se concibió la forma en que debía desarrollarse el trabajo se estimaron estas circunstancias, con la intención de que los documentos fueran precisos en sus temáticas, concisos en su contenido y ordenados en su forma.

\subsection{LAS TEMÁTICAS}

La selección de los grandes temas que constituirían el objeto de cada uno de los informes dio lugar a una primera reflexión de gran calado. Se trataba de decidir de qué se iba a informar, una pregunta que exigía que su respuesta contuviera algunas de las cuestiones científicas más significativas en el estudio de las migraciones internacionales, pero también, algunas de las temáticas más sentidas desde el punto de vista de la ciudadanía, así como aquéllas más necesitadas de reconocimiento público a efectos de su regulación. Por tanto, tres pilares básicos que justifican los asuntos abordados, pero que no eximen al grupo de trabajo de responsabilidad en cuanto a los no tratados, aquellos que no figuran y que hubieran ofrecido una perspectiva más completa de la situación de la inmigración extranjera en Canarias, y que, por distintas razones, quedaron en el camino o abordados ligeramente en el seno de rótulos más 
amplios (la perspectiva de género, las políticas educativas en materia de inmigración, el asociacionismo inmigrante, etc.).

De cada uno de los informes particulares realizados se hicieron responsables dos profesionales del equipo de trabajo. Una vez elaborado cada texto de manera preliminar, el resto de investigadores efectuaban aportaciones y expresaban su opinión al respecto, presentándose luego a la subcomisión parlamentaria el resultado del proceso científico de elaboración. Los temas abordados, organizados en documentos relativamente sintéticos, son los que se enumeran a continuación:

1. La intensificación, diversificación y concentración espacial de la inmigración y sus especificidades insulares

2. El análisis comparativo de la dinámica reciente de la inmigración en Canarias en el marco del Estado español y de las regiones europeas

3. La gestión de los flujos migratorios en una región frontera: afluencia de personas indocumentadas y control de fronteras

4. La situación específica de los menores extranjeros no acompañados

5. El significado y las implicaciones de la vertiente irregular de la inmigración

6. Los efectos de la inmigración en el mercado laboral y el papel de la economía sumergida

7. La incidencia de la inmigración en los servicios públicos fundamentales: ámbito sanitario, vertiente educativa, esfera asistencial

8. El alcance del proceso de integración y sus principales obstáculos

9. La percepción social y el discurso público sobre la inmigración

10. La gestión de la inmigración y la coordinación de las políticas públicas

11. La perspectiva territorial de la inmigración

Particularmente significativa fue la aportación geográfica, tanto en la definición inicial del catálogo de temas que se ha comentado, como en la elaboración de informes específicos, quedando bajo su responsabilidad aquéllos que tenían una importante componente socio-territorial. Se partía, en este sentido, de la experiencia adquirida por la Geografía canaria durante varias décadas de estudio de los fenómenos migratorios en el archipiélago y, concretamente, de la inmigración en la etapa más reciente de su intensificación, diversificación y extensión al conjunto de la región. De este modo, se atendió de modo particular a la realización de cinco de los documentos antes citados, que pasan a comentarse, con algo más de detalle, en sus rasgos más sobresalientes.

El primer informe que se consideró necesario realizar, en clave de contextualización general, versó sobre la dinámica geodemográfica reciente de la inmigración, en particular, sobre la intensificación, diversificación y concentración espacial de los flujos, atendiendo a las especificidades insulares. Dicho informe fue titulado $\mathrm{La}$ actual dimensión de los fenómenos migratorios en Canarias y el significado de la inmigración, basándose en la información procedente de la explotación estadística del padrón y de la estadística de variaciones residenciales, que articularon el discurso 
acerca de la población stock de procedencia extranjera y de los flujos de inmigración. La caracterización de estas dos magnitudes en una perspectiva temporal y espacial, garantizó la definición de los rasgos más significativos de la inmigración exterior de Canarias en la etapa reciente, y sirvió, por tanto, para superar prejuicios derivados de su consideración como "invasión", "avalancha", "competencia por el empleo", entre otros, que tan frecuentemente se reproducían cuando se informaba o debatía en relación con la inmigración.

El informe titulado El análisis comparativo de la dinámica reciente de la inmigración en Canarias en el marco del Estado español y de las regiones europeas pareció fundamental para situar la inmigración en su contexto geográfico y políticoadministrativo. Sólo desde un análisis comparativo podía estimarse la dimensión y la problemática específica asociada a la intensidad de los flujos de inmigración exterior en un territorio fragmentado y fronterizo, cual es el de las Islas Canarias. Por otra parte, también era preciso valorar la incidencia de este fenómeno en relación con un marco jurídico, el de la pertenencia al Estado español y al ámbito supranacional de la Unión Europea, lo que otorga un estatus peculiar a ciertas regiones comunitarias con las que guarda mayor afinidad el archipiélago, reconociéndoles un tratamiento diferenciado (ultraperiferia, regiones insulares).

La gestión de los flujos migratorios en una región frontera: afluencia de personas indocumentadas y control de fronteras fue el título sugerido para el tercer informe, ineludible en el sentido de prestar una especial atención a la arribada de personas en embarcaciones no autorizadas, que habían sobrevivido a largas y muy duras travesías, así como a la dificultad que representaba la gestión de dicha afluencia, máxime si se tiene en cuenta que la respuesta política de los gobiernos español y europeo era la de endurecer el acceso y externalizar las fronteras, dejando el control de la emigración irregular en manos de los países de origen o de tránsito. Además, las medidas de contención de flujos migratorios en relación con nuevas estructuras policiales, en especial FRONTEX y CCRC, y la ayuda de dispositivos tecnológicos cada vez más sofisticados, junto a la puesta en funcionamiento de medios de ayuda humanitaria ante situaciones de emergencia, se convertían en la cara y la cruz de un proceso de complejas implicaciones.

Es tal el grado de importancia que ha tenido la información sobre la inmigración en el marco ideológico de la ciudadanía (de qué y cómo se ha informado), así como las declaraciones públicas por parte de representantes políticos en relación con este asunto, que pareció oportuno dedicar un informe a La percepción social y el discurso público sobre la inmigración, el noveno de los realizados según el índice anterior. Las fuentes que permitieron elaborar un diagnóstico acerca del sentimiento y las actitudes frente a la inmigración procedieron del Eurobarómetro, las encuestas europeas sobre racismo y xenofobia, el barómetro de opinión del CIS, la encuesta de actitudes ante la inmigración y la integración de los inmigrantes en Canarias, más una selección de prensa local. Con todo ello, y tras el análisis pertinente, se sugirieron una serie de recomendaciones, diferenciadas en tres capítulos: el de los medios de comunicación, coincidentes con las efectuadas por ciertos Consejos Audiovisuales y colectivos de periodistas y analistas; las vinculadas a los representantes políticos; y las que pueden 
derivarse de la voz de los propios inmigrados, conociendo lo que dicen acerca del trato que se les dispensa, de la relación que mantienen con la población autóctona y con otros residentes foráneos, sean o no de su mismo origen geográfico o étnico.

El último de los informes presentados caracteriza la inmigración extranjera en Canarias desde distintas escalas de análisis, lo que le otorga un carácter eminentemente geográfico. Trata de poner de manifiesto las diferenciadas pautas de comportamiento de la inmigración desde el punto de vista territorial, de ahí su denominación: La perspectiva territorial de la inmigración extranjera. En él se estableció una categorización de los espacios de la inmigración desde el punto de vista de las procedencias y edad de los residentes extranjeros, así como del impacto diferenciado que ello representaba en los espacios insulares: municipios y distritos. Las principales conclusiones que se alcanzan tienen que ver con el hecho de que la localización de la inmigración extranjera en Canarias se ha de interpretar en función del dinamismo de los mercados de trabajo, desde una perspectiva mesoescalar (islas y municipios), y en función de pautas residenciales, desde una perspectiva microescalar (distritos y entidades de población). Por ello, propone evaluar las pautas residenciales en relación con la tendencia a la segregación o exclusión, factores que han de ser corregidos en el diseño de las políticas de integración, así como en la adecuada planificación de servicios.

Junto a esta labor de elaboración plena de informes, de evidente dimensión geográfica, los geógrafos colaboraron en los aspectos socio-territoriales del resto de los textos elaborados, particularmente en aquéllos que suponían el estudio de algunas de las implicaciones de la inmigración en la planificación de los servicios públicos fundamentales, la definición y coordinación de las políticas públicas o el desarrollo de los procesos de integración. Ello viene a reflejar la amplia perspectiva del análisis geográfico en relación a las migraciones y su relevancia, tanto de forma autónoma como cuando dicho análisis se combina con la visión de científicos sociales con los que se comparten temas de estudio y preocupaciones intelectuales. Del trabajo realizado se obtuvo además un notable enriquecimiento profesional, en la medida en que se pudieron discutir aspectos de la máxima relevancia científica con otros investigadores, así como valorar cuál podría ser su mejor proyección para a favorecer el debate político que culminara con recomendaciones para una más adecuada gestión del hecho estudiado.

\subsection{LA ESTRUCTURA DE LOS INFORMES}

La organización de la información obtenida sobre cada tema, para su entrega a la subcomisión parlamentaria, así como para la preparación de su exposición y debate posterior, fue un importante objeto de reflexión una vez que se concretó la colaboración con el Parlamento. Evidentemente se tenía que hacer siguiendo un esquema que primara lo relevante, evidenciara las cuestiones clave y destacara las propuestas. De este modo, se llegó a la siguiente estructura: 1) título, 2) planteamiento conceptual, 3) fuentes de información, 4) descripción y análisis, 5) consecuencias, implicaciones 
y problemas, 6) elementos fundamentales para la reflexión y el debate, 7) recomendaciones, propuestas y medidas, 8) dimensión europea, 9) bibliografía y 10) anexos.

Además del título pareció oportuno iniciar los correspondientes informes con una referencia al marco de análisis que condicionaba el estudio, tanto desde el punto de vista del ámbito conceptual de referencia, en función del nivel de evolución de la ciencia, como desde la perspectiva de los objetivos que trataban de alcanzarse, es decir, con una declaración de intenciones preliminar. El siguiente capítulo, dedicado a las fuentes de información, permitía argumentar que la solidez del razonamiento descansaba en la información acopiada y en sus virtudes y defectos. La descripción y el análisis eran, casi siempre, el capítulo más extenso y contaba con ilustraciones (tablas estadísticas, mapas, fotografías, gráficos) que trataban de mostrar algunos de los elementos más destacados de la exposición que se realizaba. Las consecuencias que derivaban el análisis anterior, eran tratadas en extenso en el quinto apartado, de forma que de ellas se conducía al lector hacia los elementos fundamentales que guiaban la reflexión y que fijaban los términos en que se desarrollaría el debate. Al margen de la interacción que pudiera surgir de éste, el propio informe contenía recomendaciones en forma de propuestas o medidas, que también trataban de orientar el intercambio de pareceres y que le daban una importante dimensión propositiva al trabajo.

Por otra parte, para desarrollar las ideas en un ámbito más extenso, que preocupaba especialmente a los parlamentarios, pues podían utilizar algunas de aquellas propuestas en el seno de ciertas comisiones de trabajo de la Unión Europea, se le otorgó un tratamiento especial a la dimensión europea que podían alcanzar las temáticas abordadas. Tras este apartado, se listaban las referencias bibliográficas y concluía el documento con un anexo en el que se exponían diversos paneles de indicadores. En estos paneles se respetaba la siguiente estructura, en formato de cuadro: título, denominación, fuente, entidad, periodicidad, unidad de análisis, objetivo, información, representación, resultado y comparativa, todo lo cual permitía que el lector pudiera documentarse ampliamente o, por el contrario, seleccionar los apartados que consideraba estrictamente necesario conocer, según sus intereses o responsabilidades.

Con este planteamiento se consiguió un mayor acercamiento a los destinatarios del informe, difícil en muchos casos por la distancia existente entre lo que produce habitualmente la academia y lo que necesita la praxis política. En esta oportunidad, la síntesis sirvió de aliciente para que la documentación fuese revisada antes de los debates conjuntos, y además, facilitó la elaboración del documento final, que fue recogido como texto de referencia en el Boletín Oficial del Parlamento de Canarias con el título de Informe sobre la situación de la inmigración en Canarias.

\subsection{EL DEBATE CON LOS AGENTES PÚBLICOS}

Durante los tres años en que se desarrolló la labor de redacción de informes y asesoramiento, se sucedió el siguiente procedimiento de trabajo de forma periódica, aunque condicionada por la propia agenda parlamentaria. Se entregaba el informe o los informes en la sede del Parlamento, los recibían los miembros de la subcomisión, en 
la que estaban representadas dos personas por grupo político y de entre los que una de ellas ostentaba el cargo de presidente 5 . Una vez distribuidos se fijaba una fecha para que comparecieran los miembros redactores junto a los coordinadores del informe ante la citada subcomisión, para proceder a su exposición y debate. Tras la explicación inicial, se procedía a incorporar sugerencias, si era necesario, revisándose el plan de trabajo subsiguiente: nuevas fechas de entrega, nuevo calendario de comparecencias, elaboración de documentos complementarios, etc.

En dos ocasiones se alteró dicho plan de trabajo porque la subcomisión estimó conveniente realizar más actuaciones a solicitud del grupo de trabajo, complementarias del estudio de la inmigración extranjera. Nos referimos, por una parte, a la convocatoria de reunión que se realizó al pleno del Foro Canario de la Inmigración. Dicho encuentro, en el que también participaron miembros del equipo de expertos, fue enormemente fructífero, porque permitió difundir entre estos actores sociales el contenido de la mayor parte de los informes hasta ese momento realizados y pulsar su opinión, especialmente la de los colectivos de inmigrantes y las de organizaciones no gubernamentales en él representadas.

Por otra parte, la subcomisión parlamentaria también se dirigió al Cuerpo Consular con la intención de recabar su opinión acerca de los problemas que afectaban a sus conciudadanos y de las reclamaciones que manifestaban siguiendo ese cauce, como representantes de los residentes extranjeros instalados en el archipiélago. A esta reunión también fueron invitados los miembros del grupo de trabajo, lo que permitió un mejor conocimiento de aquellos asuntos más sentidos por los inmigrados a través de la representación oficial de sus países de origen. Por motivos de agenda no pudo concretarse finalmente la comparecencia de los responsables de la Delegación del Gobierno en Canarias.

El procedimiento de trabajo escogido fue enormemente participativo, lo que garantizaba, en cierta medida, el éxito de la iniciativa y su proyección posterior al conjunto de actores y organizaciones implicadas. La culminación de este proceso fue la aprobación del documento o informe final en sede parlamentaria, tras la exposición argumentada de los distintos grupos políticos allí representados, manifestando su apoyo unánime al texto anteriormente consensuado.

\subsection{LA ELABORACIÓN DE UNA SÍNTESIS PROPOSITIVA}

Cada uno de los informes presentados junto al resultado de los debates subsiguientes con los parlamentarios y las opiniones del Foro Canario de la Inmigración y del Cuerpo Consular, en relación con el trabajo desarrollado, orientaron la redacción de la versión definitiva del informe final. En éste se prescindió de la estructura escogida para los informes monográficos previos y se ordenó con 1) un planteamiento inicial, a

\footnotetext{
${ }^{5}$ Los grupos parlamentarios que conformaron la Subcomisión, en el marco de actuación de la Comisión de Asuntos Europeos y Acción Exterior, para la elaboración de un informe sobre el trabajo realizado por las Universidades canarias sobre la situación de la inmigración en Canarias fueron el GP Socialista Canario, GP Coalición Canaria y el GP Popular.
} 
modo de declaración de intenciones; 2) un contexto, en el que se enmarcaba la elaboración del documento final, y que, además, hacía referencia a la relación que guarda el estudio de la inmigración con la importancia de este fenómeno en Canarias y con la percepción que tiene de él la sociedad regional; 3) el plan de trabajo desarrollado y los materiales obtenidos, todo ello vinculado con las temáticas abordadas; 4) la dimensión regional y europea del trabajo desarrollado; y 5) los resultados principales: recomendaciones, propuestas y medidas.

Este último capítulo corresponde al verdadero meollo del documento elaborado. No se trata de una simple adición de las recomendaciones específicas de cada uno de los informes parciales, sino de una nueva presentación, en la que se ordenaron dichas propuestas en diez apartados, para su más adecuada estimación por parte de la subcomisión que les daría traslado al Pleno del Parlamento ${ }^{6}$. Estos apartados, como conceptos clave, recibieron la denominación de conocimiento, estrategia, planificación, gestión, coordinación, control, regulación, participación, inclusión y sensibilización. Consideramos que todos ellos podían convertirse en orientaciones estratégicas, tanto para la acción de los grupos políticos como para la relación entre el Parlamento y el resto de actores públicos y privados que tienen que ver de un modo u otro con la inmigración y sus implicaciones.

A continuación, se sintetizan los contenidos más destacados de cada uno de estos epígrafes, para que pueda estimarse el interés que tienen en relación con el objeto de este artículo:

a) Conocimiento. Los análisis con enfoque escalar y suficiente nivel de desagregación informativa, los estudios comparados, las investigaciones relativas a colectivos específicos o a aspectos particulares de la inmigración, entre otras, suponen iniciativas necesarias, en las que, además, debe ser creciente la implicación de los propios protagonistas de la movilidad. El conocimiento obtenido ha de ser difundido por distintos medios, siendo las buenas prácticas, objeto de atención especial.

b) Estrategia. En la medida en que Canarias constituye una línea de fractura socioeconómica en el Atlántico, puede convertirse en un referente en materia de gestión de flujos migratorios, para lo cual se hace necesario actualizar y renovar el Pacto Canario sobre Inmigración suscrito en 2002. Ésta puede ser la base a partir de la cual se debe avanzar en el establecimiento de alianzas duraderas que permitan enfrentar con garantías la gestión de un fenómeno que ha aumentado en complejidad a lo largo del tiempo.

c) Planificación. El desarrollo de los procesos de integración y las modificaciones que ha experimentado la propia sociedad de acogida, han de ser los pilares que cimenten un nuevo diálogo social. El refuerzo de las estructuras asistenciales primarias, educación y sanidad, así como la atención a las políticas sectoriales, en particular en lo que se refiere a los mercados de trabajo y vivienda, deben constituir ejes motrices en la planificación de la atención a la nueva diversidad.

${ }^{6}$ El Pleno parlamentario en el que se presentó el informe definitivo se celebró el día 17 de enero de 2011, resultando la aprobación del mismo por unanimidad de los parlamentarios presentes. 
d) Gestión. La participación activa de la Comunidad Autónoma de Canarias en la gestión de la inmigración requiere de una intervención en dos niveles distintos. Por una parte, en la estructura administrativa propia, para la mejora del diseño de programas específicos de cara a la inmigración y, por otra, en la coordinación de los distintos agentes con competencias en inmigración (administraciones local, autonómica y estatal), redefiniendo sus recursos, mecanismos e instrumentos de actuación.

e) Coordinación. Los representantes canarios deben promover un profundo debate sobre inmigración en ciertos foros europeos como el grupo de las regiones ultraperiféricas de la Unión Europea, la comisión de islas de la CRPM y el forum de asambleas regionales europeas, con la finalidad de contribuir a diseñar una política comunitaria que responda a los retos transnacionales de la inmigración. En el seno del Estado, por otra parte, han de armonizarse los planes de inmigración de las distintas comunidades autónomas y han de elaborarse protocolos comunes con el concurso del gobierno central.

f) Control. Los dispositivos de prevención de la inmigración irregular en las fronteras exteriores de la Unión Europea y de otro tipo de desplazamientos (buques, mercancías) deben actuar con sujeción a normas respetuosas con la vida y dignidad humanas, para evitar que las fronteras se conviertan en un espacio de violencia. Por ello, se ha de avanzar hacia un modelo de gestión de fronteras más tecnológico, al mismo tiempo que se han de potenciar los medios de salvamento, de forma que las operaciones disuasorias de la inmigración irregular no impidan las de asistencia humanitaria.

g) Regulación. No es posible hablar del control de la inmigración irregular si no se adoptan medidas para potenciar ese mismo flujo de modo ordenado y legal, y además, para evitar, internamente, la economía informal. Se han de ensayar distintos mecanismos, como los cupos de mano de obra según diferentes sectores y niveles de cualificación, la reglamentación del trabajo estacional, los visados provisionales, la contratación en origen, etc. En tiempos de crisis, además, se han de redoblar los esfuerzos para evitar el avance de la contratación irregular de inmigrantes, y para que la competencia frente al empleo entre trabajadores autóctonos e inmigrados no se convierta en motivo de confrontación social.

h) Participación. Sin el concurso de las personas que han inmigrado, los planes de actuación diseñados para favorecer los procesos de integración social están llamados al fracaso. Entre las líneas de mayor éxito en este terreno se halla el mundo asociativo. La incorporación de los inmigrados en la sociedad civil también la facilitan otras vías de participación social, como las asociaciones vecinales, las asociaciones de madres y padres de alumnos, las asociaciones culturales, etc. En todas ellas debe fomentarse su presencia activa. El instrumento institucional más importante para promover su integración social es el Foro Canario de la Inmigración, que ha de adquirir una mayor autonomía, para que cumpla su cometido de vehículo de participación de los inmigrantes a través de sus asociaciones y de toda una serie de entidades relacionadas con la inmigración.

i) Inclusión. Las situaciones de exclusión de los inmigrantes impiden la cohesión de la sociedad y acentúan la vulnerabilidad de ciertos grupos humanos. Por ello, es necesario prevenir estas circunstancias con distinto tipo de políticas activas, sobre todo en materia de empleo y vivienda, articulando, en el primer caso, medidas públicas y privadas para fomentar la empleabilidad y recolocar a los trabajadores que pierden su 
empleo en situación de crisis económica y, en el segundo caso, poniendo en práctica una política que garantice el acceso de los inmigrados al mercado de la vivienda.

j) Sensibilización. Los sentimientos, actitudes y valores de los grupos sociales son tan o más importantes para la convivencia que cualquier otra actuación de carácter material o práctico. Por ello, se han de priorizar los proyectos que contribuyan a asumir la creciente multiculturalidad de nuestra sociedad, la llamada pedagogía de la diversi$\mathrm{dad}$, favoreciendo los programas de gestión y mediación intercultural y de defensa de la igualdad de género. Ahora bien, en la construcción de la opinión pública, el papel principal lo desempeñan los medios de comunicación social y el discurso de los representantes públicos, lo que exige que estos sigan códigos deontológicos ya elaborados para estos cometidos. También se han de fomentar las campañas de sensibilización en las áreas de origen, para disuadir a los candidatos a la emigración irregular por vía marítima, así como para situar en sus correctos términos las ventajas y dificultades inherentes a las migraciones internacionales.

\section{CONCLUSIONES}

En contadas ocasiones los científicos sociales tienen la oportunidad de participar en un proceso que transforme su conocimiento, producido a partir de investigaciones rigurosas y metódicas, en documentos que puedan servir como referencia para las instituciones que deben legislar y gestionar fenómenos de trascendencia social. Y menos frecuente aún es el poder discutir los resultados de su tarea con los protagonistas de dichas organizaciones, antes de convertirlos en recomendaciones o medidas concretas. Y es que la labor académica y la praxis política no se encuentran e interactúan de forma habitual, salvo casos excepcionales, como el que ha querido presentar este trabajo, que recoge la experiencia mantenida entre el Parlamento de Canarias y las universidades de La Laguna y de Las Palmas de Gran Canaria entre los años 2008 y 2010 .

La realización de un informe sobre la situación de la inmigración en Canarias se convirtió en un ejercicio enormemente sugerente para sus protagonistas, en el que la academia y la esfera parlamentaria se encontraron a partir de un objetivo común: un marco de conocimiento para facilitar la reflexión, el debate y la toma de decisiones en lo que concierne a uno de los fenómenos de mayor repercusión en las distintas dimensiones de la realidad del archipiélago a lo largo de su historia, la inmigración extranjera. Desde la definición de los temas hasta el planteamiento del procedimiento de comparecencias, pasando por el esquema de los informes que se debían elaborar, la iniciativa desarrollada ha sido original e inédita, en lo que se refiere a la actividad parlamentaria y universitaria de la región. También ha sido enormemente enriquecedor el enfoque interdisciplinar, así como de gran utilidad el acudir a los recursos científicos de que disponen las universidades.

La Geografía ha desempeñado un significativo papel en el éxito de dicha experiencia, tanto en su concepción como en su desarrollo, poniendo en valor su trayectoria de años en el estudio de las migraciones internacionales. Particularmente positiva ha sido su visión de la ciencia como instrumento de análisis en temas de honda preocu- 
pación y evidente proyección social, sobre todo con la perspectiva de que el carácter multidimensional de asuntos como las migraciones y sus implicaciones requieren un tratamiento conjunto y coordinado, desde distintos campos del conocimiento científico. Consideramos que de este tipo de iniciativas sale reforzada la disciplina y las personas que la cultivan, y que esta experiencia debe servir de ejemplo de la capacidad de liderazgo y de respuestas de dicho saber ante los grandes retos de la sociedad actual, una capacidad que la erige en un adecuado instrumento para aunar academia y praxis en asuntos decisivos para la convivencia y la cohesión de la sociedad.

\section{BIBLIOGRAFÍA}

AA.VV. (2003): Población e Inmigración en Canarias. Diagnóstico. Las Palmas de Gran Canaria. Presidencia del Gobierno de Canarias.

AA.VV. (2003): Población e Inmigración en Canarias. Conclusiones y propuestas. Las Palmas de Gran Canaria. Presidencia del Gobierno de Canarias.

BEAUJEU-GARNIER, J. (1966): Geography of Population. New York. St. Martin's Press.

BURRIEL, E. (2002): Análisis geodemográfico y planificación territorial en España. Estudios Geográficos, LXIII, 248-249, 443-469.

CAPEL, H. (2001): Dibujar el mundo. Borges, la ciudad y la geografía del siglo XXI. Barcelona, Ediciones del Serbal.

CLARKE, J. (1981): Population geography. Oxford, Pergamon Press.

CLARKE, J. (comp.) (1984): Geography and Population: Approaches and applications. Oxford. Pergamon Press.

DEMKO, GEORGE, J., HAROLD, M. ROSE \& SCHNELL, GEORGE, A. (1970): Population Geography: a reader. New York. McGraw-Hill.

FINDLEY, A. (1993): Population geographies for the 21st century. Scottish Geographical Journal, 119, 3, 177-190.

GEORGE, P. (1965): Géographie de la population. Paris. Presses universitaires de France.

GOZÁLVEZ, V. (1991): Notas sobre el estado actual de la investigación en geografía de la población. Cuadernos de Geografía, 50, 215-225.

GOZÁLVEZ, V. (1998): Notas sobre el valor educativo de la geografía de la población. IV Jornadas de Didáctica de la Geografía. Alicante.

JONES, H. (1981): A Population Geography. London, Harper \& Row Publishers.

LÉVY. J. (2011): Actores, objetos, entornos: inventar el espacio para leer el mundo. En Lindón, A. e Hiernaux, D. (Dir.): Los giros de la Geografía Humana. Desafíos y horizontes. Barcelona, Anthropos.

LINDÓN, A. e HIERNAUX, D. (Dir.) (2011): Los giros de la Geografía Humana. Desafíos y horizontes. Barcelona, Anthropos. 
MENDOZA, C. (2010): Geografía de la población: Cuantitativos versus teóricos o la sociodemografía espacial ante el desafío de nuevas propuestas teóricas y metodológicas. Cuadernos de Geografía. Revista Colombiana de Geografia, 19, 9-25.

NASH, A. (1994): Population Geography. Progress in Human Geography, 18, 385395.

NOIN, D. (1979): Géographie de la population. Paris. Armand Colin.

PACIONE, M. (1999): Applied geography: in pursuit of useful knowledge. Applied Geography, 19, 1-12.

PLANE, D. (2004): The post-Trewartha boom: the rise of demographics and applied population geography. Population, Space and Place, 10, 4, 285-288.

PARLAMENTO DE CANARIAS (2011): Informe sobre la situación de la inmigración en Canarias. Boletín Oficial del Parlamento de Canarias, 14 de enero. (http:// www.parcan.es/pub/bop/71/2011/017/bo017.pdf) [acceso 28-06-2011]

PETERS, G. \& LARKIN, R. (1979): Population Geography: Problems, Concepts and Prospects. Dubuque, IA., Kendall/Hunt Publishing Company.

RAMOS, M. y GODENAU, D. (2006): Canarias antes la inmigración africana. El País, 13 de noviembre, 17.

REQUES, P. (2011): El factor D. Los nuevos retos demográficos de la España actual. Bilbao. Cinco Días.

VINUESA, J. (1995): Análisis geodemográfico y planeamiento urbanístico. Estudios Geográficos, LVI, 219, 147-168.

WHITE, P. y JACKSON, P. (1995): (Re)theorising population geography. International Journal of Population Geography, 1, 2, 111-123.

ZELINSKY, W. (1966): A prologue to Population Geography. Englewood Cliffs, Prentice-Hall. 